\title{
Dynamic CT Imaging of Volumetric Changes in Pulmonary Nodules Correlates with Physical Measurements of Stiffness
}

\author{
Frederick M Lartey, $\mathrm{PhD}^{\mathrm{a}^{\star}}$ \\ Marjan Rafat, $\mathrm{PhD}^{\mathrm{a}^{*}}$ \\ Mohammadreza Negahdar, $\mathrm{PhD}^{\mathrm{a}}$ \\ Andrey V Malkovskiy, $\mathrm{PhD}^{\mathrm{b}}$ \\ Xinzhe Dong, MD ${ }^{\text {ad }}$ \\ Xiaoli Sun, MD ${ }^{\text {ae }}$ \\ Mei Li, MD ${ }^{\text {af }}$ \\ Timothy Doyle, DPhil. ${ }^{\mathrm{c}}$ \\ Jayakumar Rajadas, $\mathrm{PhD}^{\mathrm{b}}$ \\ Edward E Graves, $\mathrm{PhD}^{\text {ag }}$ \\ Billy W Loo, MD, PhD ag• \\ Peter G Maxim, PhD ${ }^{\text {ag }}$
}

${ }^{a}$ Department of Radiation Oncology, Stanford University School of Medicine, Stanford, CA

${ }^{\mathrm{b}}$ Biomaterials and Advanced Drug Delivery Laboratory, Cardiovascular Pharmacology Division, Cardiovascular Institute, Stanford University School of Medicine, Stanford, CA

${ }^{c}$ Department of Pediatrics, Stanford University School of Medicine, Stanford, CA

' Department of Radiation Oncology, Shandong Cancer Hospital, Shandong University, Shandong, China

e Department of Radiation Oncology, The First Affiliated Hospital of Zhejiang University, Zhejiang, China

' Department of Radiation Oncology, Cancer Hospital, Guangdong, China

${ }^{9}$ Stanford Cancer Institute, Stanford University School of Medicine, Stanford, CA

"Co-First authors: Frederick M Lartey, PhD AND Marjan Rafat, PhD

•Corresponding authors: Peter G. Maxim, Ph.D., Stanford Cancer Center, 875 Blake Wilbur Dr., Stanford, CA 94305. Tel: (650) 724-3018; Fax: (650) 725-8231; E-mail: pmaxim@stanford.edu 
Billy W. Loo, Jr., M.D., Ph.D., Stanford Cancer Center, 875 Blake Wilbur Dr., Stanford, CA 94305. Tel: (650) 736-7143; Fax: (650) 725-8231; E-mail: bwloo@stanford.edu

Funding: This study was supported by Department of Defense LCRP 2011 \#W81XWH-12-10286. MR was supported by the National Institutes of Health K99CA201304.

This paper has 13 pages, 6 figures, and 2 supplementary figures.

Running head: Imaging and stiffness of pulmonary nodules 


\section{Abstract}

Background and purpose: A major challenge in CT screening for lung cancer is limited specificity when distinguishing between malignant and non-malignant pulmonary nodules (PN). Malignant nodules have different mechanical properties and tissue characteristics ('stiffness') from non-malignant nodules. This study seeks to improve CT specificity by demonstrating in rats that measurements of volumetric ratios in PNs with varying composition can be determined by respiratory-gated dynamic CT imaging and that these ratios correlate with direct physical measurements of PN stiffness.

Methods and materials: Respiratory-gated microCT images acquired at extreme tidal volumes of 9 rats with PNs from talc, matrigel and A549 human lung carcinoma were analyzed and their volumetric ratios $(\delta)$ derived. PN stiffness was determined by measuring the Young's modulus using atomic force microscopy (AFM) for each nodule excised immediately after microCT imaging.

Results: There was significant correlation $(P=0.0002)$ between $P N$ volumetric ratios determined by respiratory-gated CT imaging and the physical stiffness of the PNs determined from AFM measurements.

Conclusion: We demonstrated proof of concept that PN volume changes measured noninvasively correlate with direct physical measurements of stiffness. These results may translate clinically into a means of improving the specificity of CT screening for lung cancer and/or improving individual prognostic assessments based on lung tumor stiffness.

Keywords: Pulmonary Nodules; Deformability; Respiratory-gated MicroCT; Lung Cancer, Rats. 


\section{Introduction}

Although advances in surgery, radiation therapy, and systemic therapies in lung cancer have improved survival, clinical stage at diagnosis remains the major determinant of survival after therapy [1]. Screening for lung cancer with low-dose computed tomography (CT) is a highly sensitive, non-invasive imaging modality that has demonstrated mortality reduction through early detection of the disease [2]. CT imaging has also been used to delineate lung lesions by deformable image registration for more accurate radiation treatment planning and therapy delivery [3-5]. However, the key challenge for CT as a screening tool is limited specificity to distinguish between malignant and non-malignant pulmonary nodules (PN) leading to a high false positive rate.

Imaging, particularly ultrasound, has improved the ability to differentiate between malignant and benign tumors based on mechanical properties in breast carcinoma [6, 7], metastatic melanoma [8, 9], head and neck carcinoma [10], and colorectal carcinoma [6]. Differences in trajectory distortion can detect the subtle changes in mechanical properties between malignant and non-malignant tumors. Ultrasound imaging, however, is technically challenging for lung tissue, due to the opacity of air in the lungs. In addition, MRI-based imaging techniques that assess tissue stiffness in hepatic fibrosis [11,12] are also susceptible to artifacts from air in the lung.

We previously developed a non-invasive CT-based imaging methodology that differentiated the volumetric changes in malignant PN from those of the surrounding lung tissue $[13,14]$. High resolution dynamic CT imaging, in contrast to static imaging, captures temporal changes in lung and PN volumes during the respiratory cycle. We hypothesize that dynamic CT images acquired at various points in the respiratory cycle such as at the extremes of tidal volume (peak-inhale and exhale) can differentiate PNs based on mechanical properties revealed as differences in volume changes of the PNs. Malignant tumors in various organs have 
higher interstitial fluid pressure rendering them 'stiffer' relative to the surrounding tissues [6-10]. Intuitively, they should undergo significantly smaller volume changes from one respiratory phase to another. Conversely, the less 'stiff,' non-malignant nodules would be expected to undergo significantly larger volume changes.

This study seeks to demonstrate that volumetric ratios in PNs with varying composition can be non-invasively determined by respiratory-gated dynamic CT imaging and that these ratios correlate with direct physical measurements of PN 'stiffness'. We used atomic force microscopy (AFM) to measure the elastic properties of malignant and benign lung lesions. Force-distance curves are generated by AFM measurements where a cantilever indents a sample and the deflection is measured to probe tissue elasticity on a local scale [15]. This technique has been used previously to determine the Young's modulus of biological tissues [1618]. The study is particularly important as it allows for comparisons of direct physical vs. noninvasive imaging measurements of pulmonary nodule properties in a preclinical model, a procedure that cannot be performed in human subjects.

\section{Materials and methods}

\section{Cell Culture}

A549 human lung carcinoma cells were obtained from ATTC (CCL-185; Manassas, VA, USA). Cells were cultured in F-12K medium supplemented with $10 \%$ FBS and $1 \%$ penicillinstreptomycin and incubated in $95 \%$ air $/ 5 \% \mathrm{CO}_{2}$ at $37^{\circ} \mathrm{C}$.

\section{Animal Models and Pulmonary Nodule Generation}

All experiments were conducted with approval by the Administrative Panel on Laboratory Animal Care (APLAC) of Stanford University. A total of 18 (9 for non-invasive imaging and AFM studies; and 9 for histological studies) RNU female rats (Taconic Biosciences, Petersburgh, NY) were anesthetized using $2 \%$ isoflurane and placed inside the Spectral Ami X Optical imaging instrument (Spectral Instruments Imaging, Tuscon, AZ). The scanner was used to facilitate high- 
precision image guidance for injection and localization within the lung. In each case, a 1 cc syringe with a $30 \mathrm{G}$ needle was introduced through the intercostal space and held in place inside the lung of the anesthetized rat. Before injection, an X-ray image was obtained to determine whether the needle was placed within the desired location (Figure 1).

Three different types of PNs were generated in the animals: (i) Talc - $20 \mathrm{mg}$ of talc/80 $\mu \mathrm{l}$ PBS (Sigma-Aldrich, St. Louis, MO) was injected into the right lung of 3 rats. (ii) Malignant - to generate orthotopic lung xenograft tumor models, 4 rats, 3 of which had been previously injected with talc in the right lung, underwent whole body irradiation (4 Gy). A total of $2 \times 10^{6}$ A549 lung cancer cells in matrigel (diluted 1:1 in PBS) in a final volume of $80 \mu$ l were then injected into the intercostal space of the left lung 24 hours after irradiation. The rats were then monitored for 60 minutes to ensure they showed no negative side effects from the procedure. (iii) Matrigel - To simulate PNs with different mechanical properties from malignant A549 lung tumors, matrigel $(80 \mu \mathrm{l})$ was injected into the right lung of another 5 rats. Injection was completed only after X-ray images confirmed the needle had been placed in the correct location within the lung. Time of imaging and AFM measurements for PNs from malignant tumors and talc were 4 weeks post inoculation and 1 hour post injection for matrigel. To account for the differences in volume sizes, volumetric ratios (normalizing over the disparate volume sizes) were used in the study, rather than the mean nodule volume.

Respiratory-Gated MicroCT Imaging and Reconstruction

Scanning was performed with an eXplore Locus RS-150 MicroCT rodent model instrument (TriFoil Imaging (Northridge, CA)) fitted with a nose cone for isoflurane anesthesia administration. The rats were anesthetized, maintained under $2 \%$ isoflurane in oxygen and subsequently placed prone on the MicroCT scanner bed with a respiratory sensor pad underneath the abdomen (Supplementary Figure 1A). X-rays were generated from a $70 \mathrm{kVp}$, $40 \mathrm{~mA}$ source with the following acquisition parameters: 400 views over an angle of increment 
of $0.7^{\circ}$, averaging 2 frames per view; exposure time: $17 \mathrm{~ms}$; resolution: $0.096 \mathrm{~mm}$; with $4 \times 4$ binning on the charge coupled device detector. The gating instrument (Spin Systems Pty Ltd., Queensland, Australia) converted pressure on the respiratory sensor pad generated during breathing into a voltage signal. The output voltage was used to trigger image acquisition on the MicroCT scanner at any desired time point in the respiratory cycle (Supplementary Fig. 1B). Peak-inhale and peak-exhale images were consecutively acquired with a temporal resolution of 10 ms using the Biovet software program (Spin Systems Pty Ltd., Queensland, Australia).

Image reconstruction was performed using eXplore MicroView (GE Healthcare, Fairfield, CT). Each scanned image was centered in a $46.25 \mathrm{~mm}$ axial field of view $(84.7 \mathrm{~mm}$ transaxial field of view). Images were first calibrated, corrected, and reconstruction was performed at an isotropic voxel size of $97.3 \mu \mathrm{m}$.

Image Analysis and Derivation of a Volumetric Ratio Parameter ( $\delta$ )

Post-acquisition nodule contouring for each of the 2 respiratory phases was performed by 4 independent observers using MIM software (MIM Software Inc., Cleveland, OH). To compensate for inter-observer variability, the average volume of the PNs delineated by the 4 observers was used in determining the volumetric change of each nodule. Nodule delineation and volume changes were determined through contouring: regions of interest (ROIs) were drawn on every slice to delineate each nodule and subsequently merged to form volumes for each tidal extreme (inhale or exhale). A volumetric ratio parameter $(\delta)$ was defined as the ratio of the volume of the PN on inhale to that at exhale (Equation 1).

$$
\delta=\frac{V_{\text {inhale }}}{V_{\text {exhale }}}
$$

where $\mathrm{V}$ represents the volume obtained from image analysis.

Histology

A set of 3 animals each with talc, matrigel or malignant PNs generated in a similar manner as previously described, were euthanized with carbon dioxide and the lungs harvested and placed 
in $4 \%$ paraformaldehyde for 24 hours. They were subsequently enclosed in a cassette and stored in $70 \%$ ethanol until they were embedded in paraffin and sectioned. Hematoxylin and eosin $(H \& E)$ staining was performed for 3 rats with each nodule type. Images were obtained using brightfield microscopy. Although all animals underwent MicroCT imaging, animals used for histology (9 in total) were different from those used for AFM measurements.

\section{Atomic Force Microscopy}

After microCT imaging, the rats were sacrificed, and the Young's modulus was determined for each nodule using atomic force microscopy (AFM). Immediately after imaging, the nodules were harvested fresh from the lungs and kept in pre-warmed PBS at $37^{\circ} \mathrm{C}$. Force-distance (FD) measurements of excised tissues were performed in a fluid cell, 2-4 hours after excision. Measurements were taken either using an Agilent 5500 Scanning Probe Microscope (Agilent Technologies, Santa Clara, CA) or a Park NX-10 AFM (Park Systems, Santa Clara, CA) when temperature control was necessary. Tips with a silicon oxide spherical indenter (950 nm radius, $k=0.08 \mathrm{~N} / \mathrm{m}$ as reported by the manufacturer) were used (NanoAndMore USA, Lady's Island, SC). The moduli were verified and reproduced on both machines for all samples with the exception of the matrigel samples, which could only be analyzed using the $\mathrm{NX}-10$ at $37^{\circ} \mathrm{C}$ in order to preserve solid gel properties. Samples were probed a minimum of 10 times in at least 3 distinct regions $20 \mu \mathrm{m}$ apart for obtaining a representative result. Young's moduli were calculated with SPIP software (Image Metrology, Hørsholm, Denmark), which used the Hertz model for spherical indenters to fit the initial indentation curve (Supplementary Figure 2).

\section{Statistical analysis}

Statistical analysis was performed using SAS 9.4 software (SAS Institute Inc., Cary NC). Pearson's correlation coefficient was determined to establish whether there was a significant degree of linear dependence between the non-invasive nodule volumetric ratios from respiratory-gated dynamic CT and their physical stiffness as determined by AFM measurement. 


\section{Results}

Image guidance for injection and localization using the Spectral Ami X Optical imaging (Figure 1) ensured successful PN establishment in the left (matrigel or A549 tumor) and right (talc) lungs (Figure 2). Mean tumor and talc sizes, obtained from CT imaging were $0.4 \pm 0.04$ and 0.3 $\pm 0.03 \mathrm{~cm}^{3}$ respectively. Mean matrigel volume was $1.6 \pm 0.10 \mathrm{~cm}^{3}$. PNs formed from talc were particularly heterogeneous with some calcified nodules identifiable on MicroCT imaging, but their volumes did not change over time. MicroCT gating (Supplementary Figure 1) ensured the acquisition of CT images only at extreme tidal volumes (Supplementary Figure 2). An overlay of the images acquired at peak tidal volumes (Figure 3) indicated that the lung volume at inhale, on average, was $16 \%$ higher than the lung volume at exhale. The mean volumetric ratios, inhale to exhale, for matrigel, malignant, and talc PNs were 1.05, 0.96 and 1.01, respectively.

To validate our non-invasive method, AFM was used to determine the stiffness of PNs. FD measurements (Figure 4A) were taken to determine the Young's moduli, and our sample positioning and representative test regions are shown in Figure 4B. The range of elastic moduli for malignant, talc, and matrigel PNs is shown in Figure 4C. The average Young's modulus for malignant PNs was significantly higher than those for matrigel and normal lung tissue but was not significantly different from talc. The mean value for the malignant PNs was $2552 \mathrm{~Pa}$, in comparison to values of 2611, 1287 and $1304 \mathrm{~Pa}$ for talc, matrigel, and normal lung tissue, respectively. Malignant PN stiffness was thus a factor of 1.98 and 1.96 higher than for matrigel and normal lung tissue, respectively. The higher stiffness of talc over matrigel and normal lung did not reach statistical significance. H\&E staining showed the microscopic appearance of the malignant, talc, and matrigel PNs in comparison to lungs from control rats (Figure 5). There was a significant correlation between the nodule volumetric ratios determined by the non-invasive imaging and physical measurements of stiffness (Figure 6).

\section{Discussion}


The present study sought (i) to determine by non-invasive imaging, volumetric ratios in PNs with varying composition and physical characteristics in a rat model of orthotopic human lung cancer and non-malignant nodules, and (ii) to determine whether the imaging-based volumetric ratios correlated with direct physical measurements of individual nodule stiffness. We demonstrated proof of principle that minute changes in $\mathrm{PN}$ volume during the respiratory cycle could be determined and analyzed, and that they correlate with physical measurements of stiffness obtained from AFM FD measurements.

Malignant solid tumors have different mechanical properties and tissue characteristics from nonmalignant tumors. Increased stress due to uncontrolled proliferation of malignant cells and the contribution of stromal cells to extracellular matrix reorganization and deposition have been shown to enhance the stiffness of the tumor microenvironment compared to benign lesions [19]. In addition, differences in mechanical properties may be attributed to increased interstitial fluid pressure in malignant tumors, as reported for breast carcinoma [6, 7], metastatic melanoma [8, 9], head and neck carcinoma [10], and colorectal carcinoma [6] with values as high as 60 $\mathrm{mmHg}$. While the underlying mechanisms have not been fully elucidated, they are thought to involve blood-vessel leakiness, lymphatic vessel abnormalities, interstitial fibrosis, and a contraction of the interstitial space mediated by stromal fibroblasts, all of which are hallmarks of cancer $[20,21]$. This results in uniform pressure throughout the center of the nodule which then drops steeply towards the periphery [22-24]. While increased interstitial fluid pressure has not been directly shown for malignant lung tumors, we hypothesized that they would exhibit similar properties.

While we previously demonstrated that dynamic CT in patients could in principle be used to determine parameters related to pulmonary nodule elasticity, there is no practical method in human patients to validate the imaging measures with a direct physical measurement of nodule stiffness. As such, we turned to a preclinical model of implanted pulmonary nodules with a 
variety of mechanical properties. An orthotopic lung tumor model using A549 cells in rats was used [25]. These cells were malignant, and the resulting tumors were expected to be stiffer than the surrounding normal lung tissue. To create a non-malignant model, matrigel, which is a solubilized basement membrane preparation extracted from mouse sarcoma and enriched with a number of growth factors [26] was injected into the rat lung. At $37^{\circ} \mathrm{C}$, matrigel forms a gel within the lung, which appears as a nodule upon CT imaging, indistinguishable from a malignant nodule (Figure 2). We hypothesized that the properties of matrigel differ from the malignant tumor nodules such that the interstitial pressure and resulting stiffness would be substantially lower. Our third animal model involved the use of talc in forming the PNs. Unlike matrigel or A549 lung cancer cells which consistently resulted in nodules with similar tissue characteristics, nodules from talc ranged from deposits of hard crystal aggregates to softer gel-like granulomas. We thus anticipated that their volumetric changes would exhibit greater variability than those for malignant or matrigel PNs. The Young's modulus of each nodule post imaging was measured ex vivo using AFM, and correlated significantly with the non-invasively determined volume ratio by dynamic CT.

A primary limitation of this study involved generating the non-malignant nodules. Clinically, benign nodules are a heterogeneous group of neoplastic, inflammatory, or other soft tissue lesions. These include but are not limited to granuloma, hamartoma, lipoma, cysts, and intrapulmonary lymph nodes [27]. We were unable to create animal models spanning all the various forms and sources of non-malignant nodules. Furthermore, the technical complexity of this experimental system limited the overall sample size that could practically be studied. A second limitation was the small sample size of the rats within each sub-group of pulmonary nodule type. However, 12 nodules of different types and mechanical characteristics were sufficient to demonstrate proof of principle for our hypothesis of correlation between nodule volume change by imaging and stiffness by AFM. 
A current limitation of CT screening for lung cancer is limited specificity that leads to a high false positive rate, prompting follow up imaging and/or invasive diagnostic procedures. Our results may be clinically translated by adding information about pulmonary nodule mechanical properties through a simple modification to existing scanning protocols. This information may improve the specificity of screening leading to earlier diagnosis or decreased invasive procedures. Furthermore, in established diagnoses of lung cancer, the magnitude of tumor stiffness might correlate with biological aggressiveness, and this non-invasive imaging technique may add individual prognostic or predictive information that may improve personalized therapy. These are concepts that can readily be tested in prospective clinical trials.

We demonstrated proof of concept that pulmonary nodule volume changes measured by noninvasive dynamic CT correlates with direct physical measurements of nodule stiffness measured by atomic force microscopy in a preclinical model. These results may translate clinically into a means of improving the specificity of CT screening for lung cancer and/or improving individual prognostic assessments based on lung tumor stiffness. Future goals involve using this methodology to distinguish malignant from benign PNs in larger animals and humans.

\section{Acknowledgements}

We would like to acknowledge the Stanford Cancer Institute and the Stanford Center for Innovation in In-Vivo Imaging $\left(\mathrm{SCl}^{3}\right)$ for support in performing the study. 


\section{References}

[1] Mountain CF. Revisions in the International System for Staging Lung Cancer. Chest 1997;111:1710-7.

[2] Aberle DR, Berg CD, Black WC, et al. The National Lung Screening Trial: overview and study design. Radiology 2011;258:243-53.

[3] Orban de Xivry J, Janssens G, Bosmans G, et al. Tumour delineation and cumulative dose computation in radiotherapy based on deformable registration of respiratory correlated CT images of lung cancer patients. Radiother Oncol 2007;85:232-8.

[4] van Dam IE, van Sornsen de Koste JR, Hanna GG, et al. Improving target delineation on 4-dimensional CT scans in stage I NSCLC using a deformable registration tool. Radiother Oncol 2010;96:67-72.

[5] Speight R, Sykes J, Lindsay R, Franks K, Thwaites D. The evaluation of a deformable image registration segmentation technique for semi-automating internal target volume (ITV) production from 4DCT images of lung stereotactic body radiotherapy (SBRT) patients. Radiother Oncol 2011;98:277-83.

[6] Less JR, Posner MC, Boucher Y, et al. Interstitial Hypertension in Human Tumors .4. Interstitial Hypertension in Human Breast and Colorectal Tumors. Cancer Res 1992;52:6371-74.

[7] Nathanson SD, Nelson L. Interstitial Fluid Pressure in Breast-Cancer, Benign Breast Conditions, and Breast Parenchyma. Ann Surg Oncol 1994;1:333-38.

[8] Boucher Y, Kirkwood JM, Opacic D, Desantis M, Jain RK. Interstitial Hypertension in Superficial Metastatic Melanomas in Humans. Cancer Res 1991;51:6691-94.

[9] Curti BD, Urba WJ, Alvord WG, et al. Interstitial Pressure of Subcutaneous Nodules in Melanoma and Lymphoma Patients - Changes during Treatment. Cancer Res 1993;53:2204-07.

[10] Gutmann R, Leunig M, Feyh J, et al. Interstitial Hypertension in Head and Neck Tumors in Patients - Correlation with Tumor Size. Cancer Res 1992;52:1993-95.

[11] Yin M, Talwalkar JA, Glaser KJ, et al. Assessment of hepatic fibrosis with magnetic resonance elastography. Clin Gastroenterol H 2007;5:1207-13.

[12] Venkatesh SK, Yin M, Ehman RL. Magnetic resonance elastography of liver: Technique, analysis, and clinical applications. J Magn Reson Imaging 2013;37:544-55.

[13] Negahdar M, Shultz DB, Eyben RV, et al. Noninvasive Pulmonary Nodule Elastometry by CT and Deformable Image Registration. Int J Radiat Oncol 2014;90:S623-S24.

[14] Negahdar M, Fasola CE, Yu AS, et al. Noninvasive pulmonary nodule elastometry by CT and deformable image registration. Radiother Oncol 2015;115:35-40.

[15] Cappella B, Dietler G. Force-distance curves by atomic force microscopy. Surf Sci Rep 1999;34:1-104.

[16] Cross SE, Jin YS, Rao J, Gimzewski JK. Nanomechanical analysis of cells from cancer patients. Nat Nanotechnol 2007;2:780-83.

[17] Lekka M, Gil D, Pogoda K, et al. Cancer cell detection in tissue sections using AFM. Arch Biochem Biophys 2012;518:151-6. 
[18] Plodinec M, Loparic M, Monnier CA, et al. The nanomechanical signature of breast cancer. Nat Nanotechnol 2012;7:757-65.

[19] Nagelkerke A, Bussink J, Rowan A, Span P. The mechanical microenvironment in cancer: How physics affects tumours. Semin Cancer Biol 2015;35:62-70.

[20] Guerrero T, Zhang G, Huang TC, Lin KP. Intrathoracic tumour motion estimation from CT imaging using the 3D optical flow method. Phys Med Biol 2004;49:4147-61.

[21] Murphy K, van Ginneken B, Reinhardt JM, et al. Evaluation of registration methods on thoracic CT: the EMPIRE10 challenge. IEEE Trans Med Imaging 2011;30:1901-20.

[22] Rietzel E, Pan T, Chen GT. Four-dimensional computed tomography: image formation and clinical protocol. Med Phys 2005;32:874-89.

[23] Kabus S, Klinder T, Murphy K, et al. Evaluation of 4D-CT lung registration. Med Image Comput Comput Assist Interv 2009;12:747-54.

[24] Kabus S, Franz A, Fischer B. Spatially varying elasticity in image registration. Methods Inf Med 2007;46:287-91.

[25] Whitehead CM, Earle KA, Fetter J, et al. Exisulind-induced apoptosis in a non-small cell lung cancer orthotopic lung tumor model augments docetaxel treatment and contributes to increased survival. Mol Cancer Ther 2003;2:479-88.

[26] Hall DM, Brooks SA. In vitro invasion assay using matrigel: a reconstituted basement membrane preparation. Methods Mol Biol 2014;1070:1-11.

[27] Suut S, Al-Ani Z, Allen C, et al. Pictorial essay of radiological features of benign intrathoracic masses. Ann Thorac Med 2015;10:231-42. 


\section{Figures}

FIGURE 1. Representative spectral AMI X image-guided implantation of cells, talc and matrigel to form pulmonary nodules.

FIGURE 2. Representative MicroCT images of the rat lung obtained at peak inhale showing pulmonary nodules formed from malignant A549 carcinoma cells (A), talc (B) and matrigel (C); The arrows denote each nodule.

FIGURE 3. (A) An overlay of respiratory-gated MicroCT images of the thoracic region of the rat at peak inhale (black and grey) and peak exhale (rainbow). (B): Schematic diagram showing the derivation of volumetric ratios from pulmonary nodules. Values were generated by contouring regions of interest (ROIs) drawn on every Micro CT image slice to delineate each nodule and subsequently added up to form a 3-dimensional volume representing the whole nodule.

FIGURE 4. Measuring the elasticity of biological tissues using atomic force microscopy (AFM). (A) Schematic of AFM using a spherical tip for FD measurements. (B) Lung nodules in the AFM sample holder and sites of modulus measurements. Samples were probed 10 times in each region for accurate measurements. (C) Young's moduli of malignant, talc, and matrigel nodules and lung tissue samples using the Hertz model. Bars represent standard error measurements.

FIGURE 5. Hematoxylin and eosin stain (H\&E staining) for pulmonary nodules derived from (A) A549 tumor cells, (B) talc, and (C) matrigel compared to (D) normal lung tissue. Scale bar is $100 \mu \mathrm{m}$.

FIGURE 6. Correlation between nodule volumetric ratio and Young's modulus for tumor (pink), matrigel (blue), and talc (green) nodules. 
*Inclusive of all data, the correlation is significant $\left(R^{2}=0.4703, p=0.013\right)$ and increases substantially exclusive of an outlier data point for talc. 


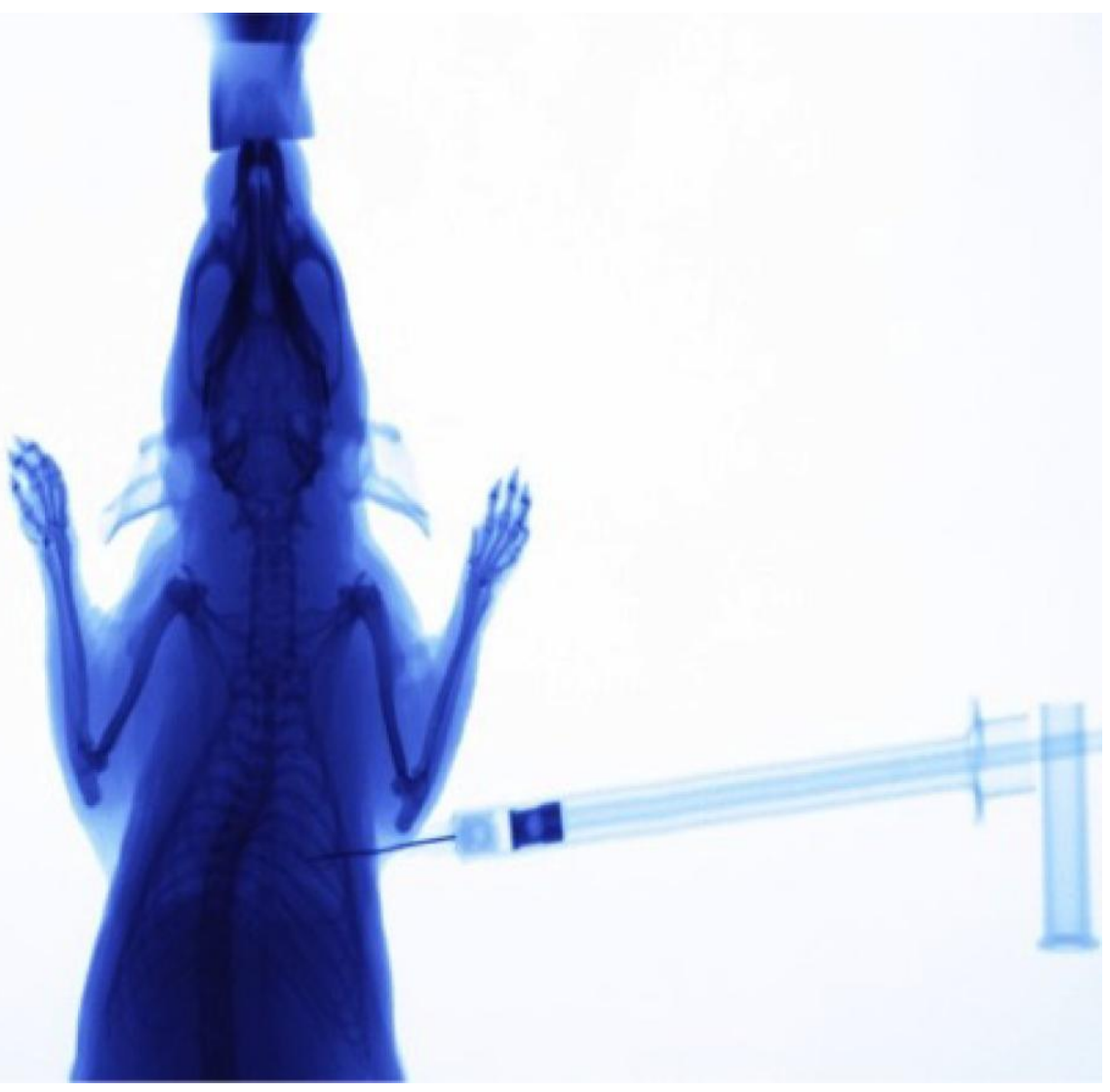




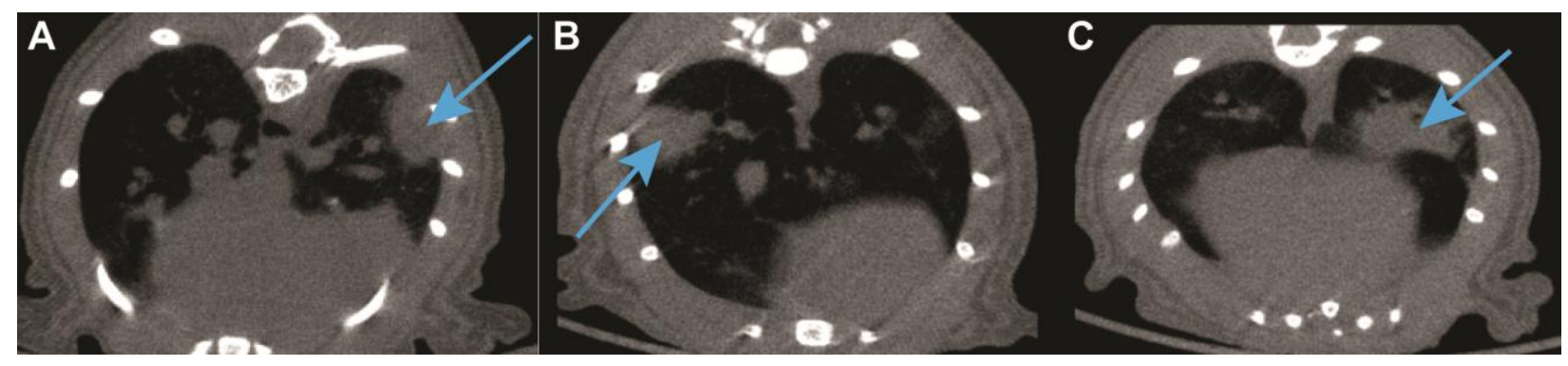


A

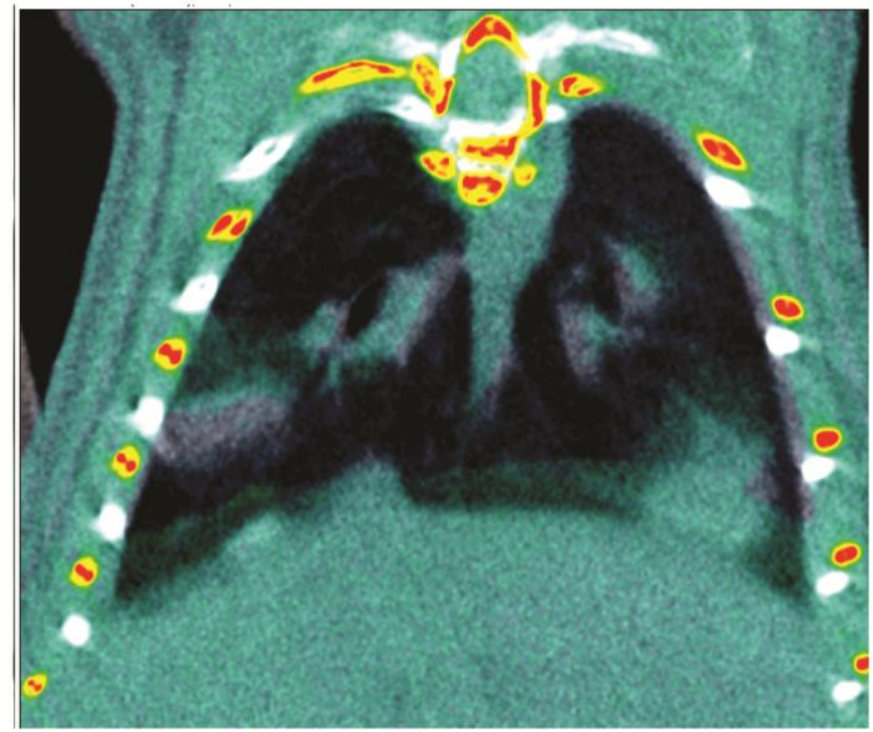

B
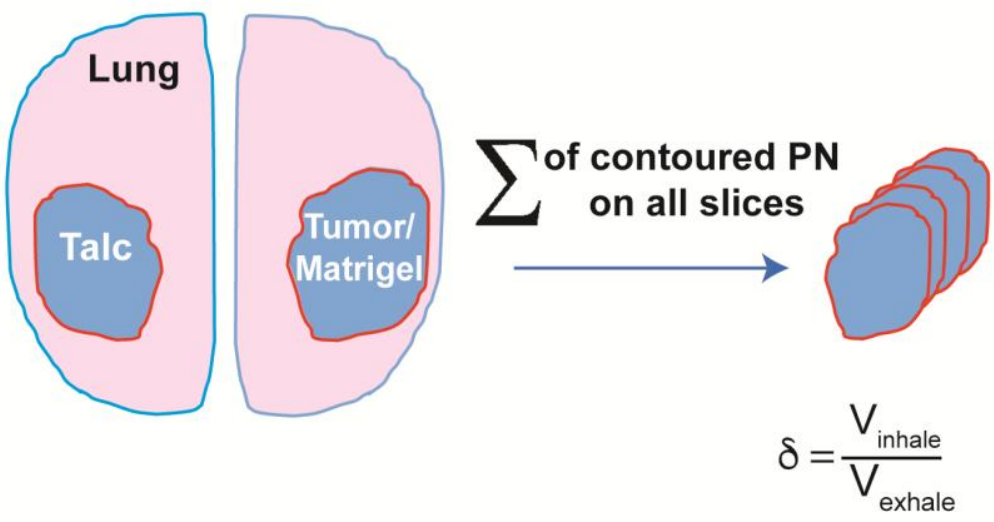

3D nodule volume, $\mathrm{V}$, at inhale or exhale

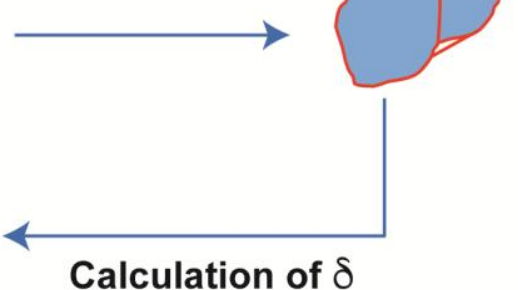


A

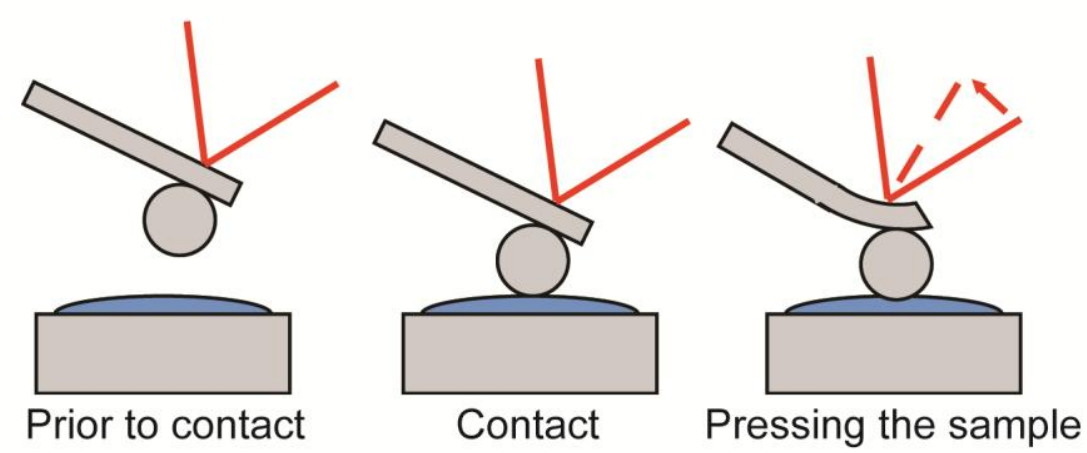

B

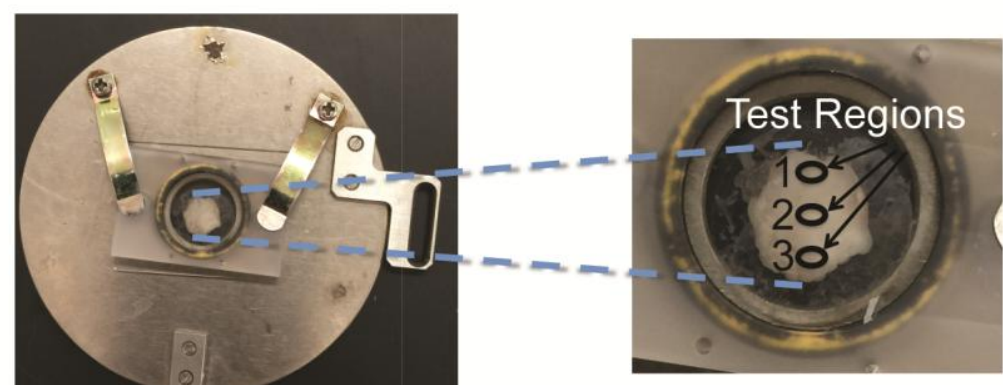

C

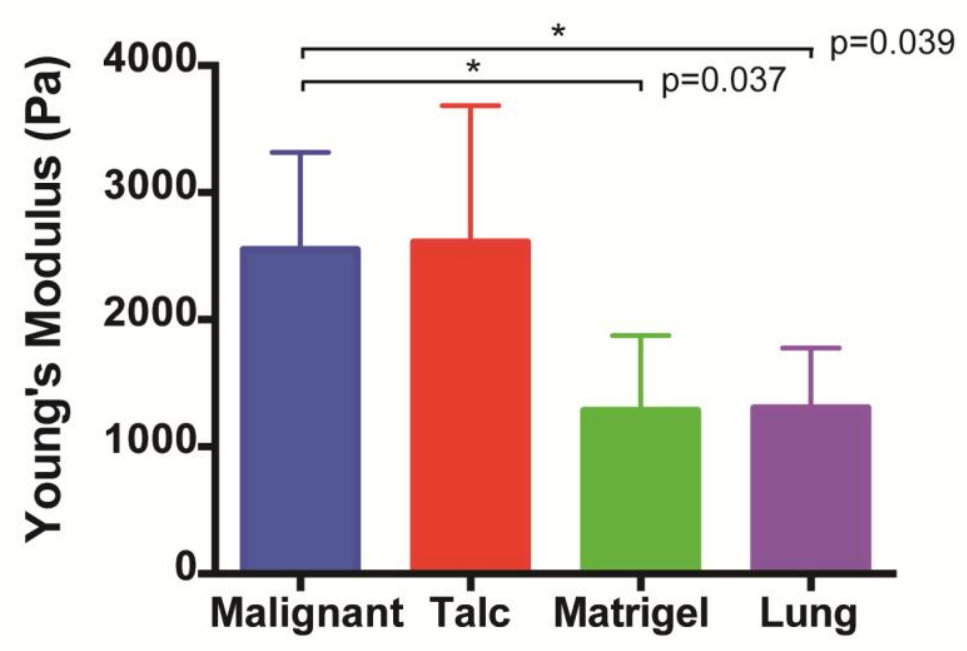



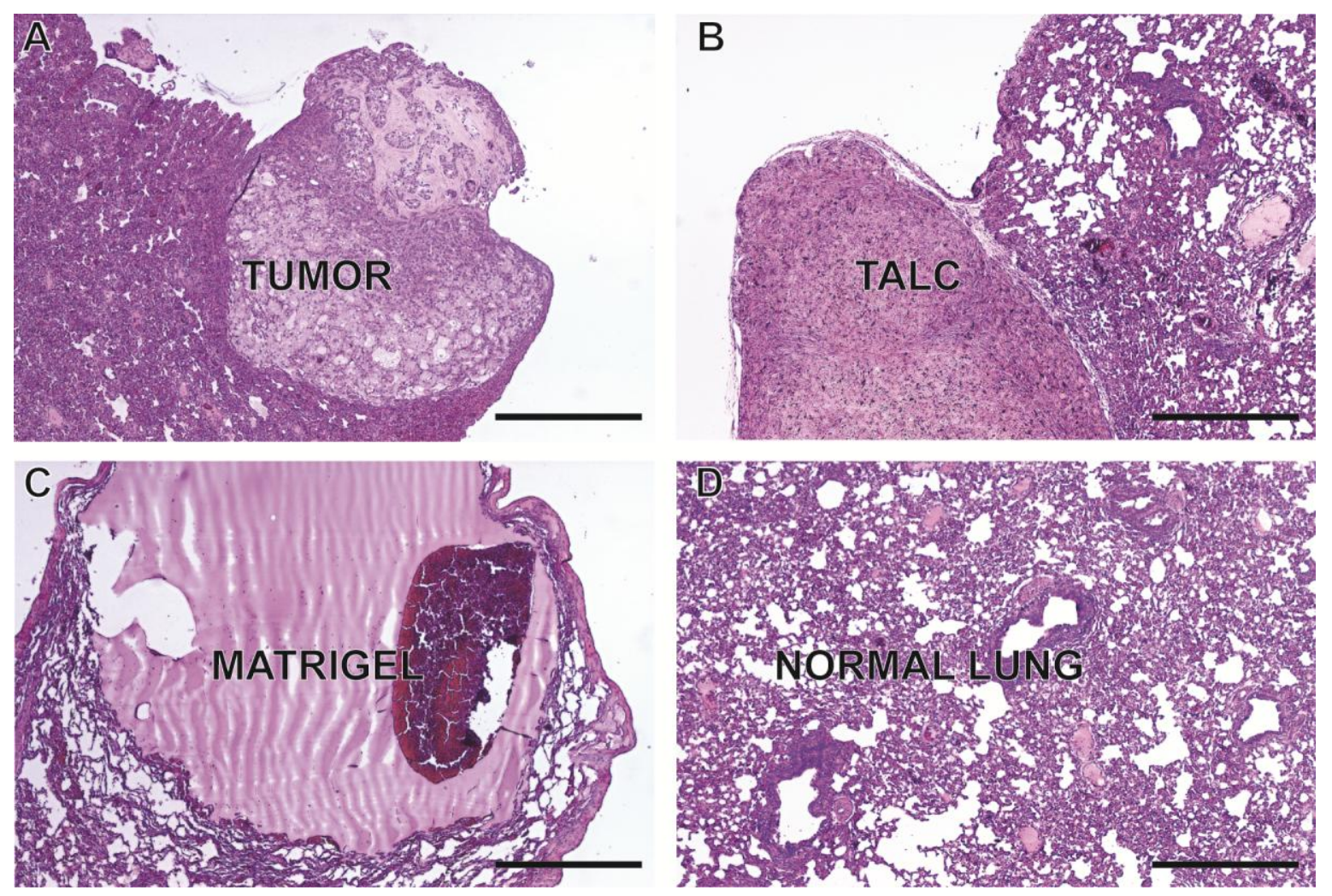


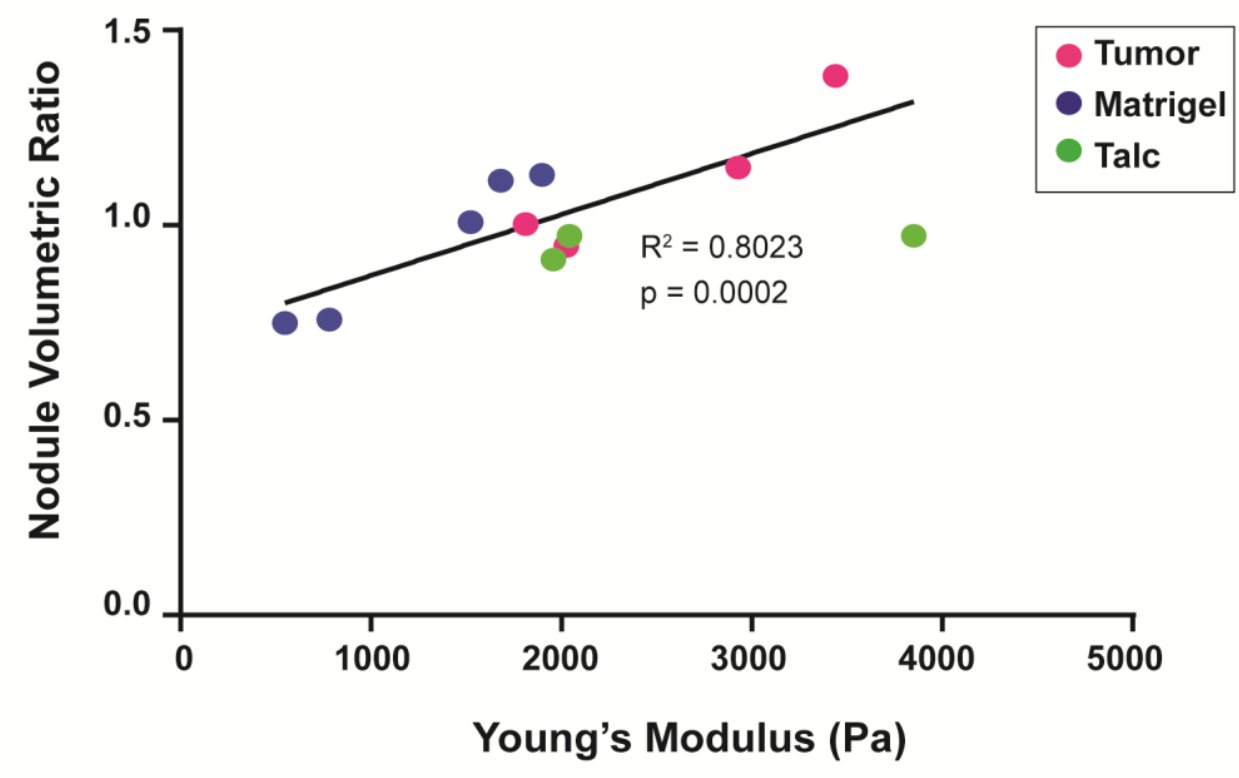

